\title{
Electronic health records as an equity tool for LGBTQIA+ people
}

\author{
Collection of data on sexual orientation, gender identity and intersex status will help to reduce health disparities \\ that affect people from sexual and gender minority communities.
}

\section{Alex S. Keuroghlian}

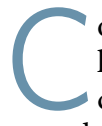
ompared to the general population, lesbian, gay, bisexual, transgender, queer, intersex, asexual and all sexual and gender minority (LGBTQIA+) communities experience a multitude of health disparities. As a result, the 2020 US National Academies of Sciences, Engineering, and Medicine Consensus Study Report, "Understanding the Wellbeing of LGBTQI+ Populations," concluded that data on sexual orientation, gender identity and intersex status are required to inform research, engage in population-level monitoring, determine resource allocation and set policies that effectively address these inequities ${ }^{1}$.

\section{How to collect the data}

Routine collection of sexual orientation and gender identity data in healthcare has high acceptability across racially and geographically diverse US communities. A study of primary care patients, most of whom were cisgender and straight, as well as mostly Black, Indigenous and people of color, reported that most respondents believed that their sexual orientation and gender identity are important for their medical provider to know, answered questions about these at registration and expressed willingness to answer them again in the future ${ }^{2}$. Some patients may have privacy concerns and benefit from understanding the confidentiality safeguards that apply to their sexual orientation and gender identity data. Transgender and gender diverse community members, as well as healthcare professionals, describe the importance of standardized gender-affirming intake forms and electronic health records (EHRs) that facilitate asking for and using each patient's correct name and pronouns ${ }^{3}$.

Healthcare organizations can adopt well-established strategies for data collection. For example, revising language for inclusivity in patient-facing materials, forms and processes will foster environments conducive to collecting data about sexual orientation, gender identity and

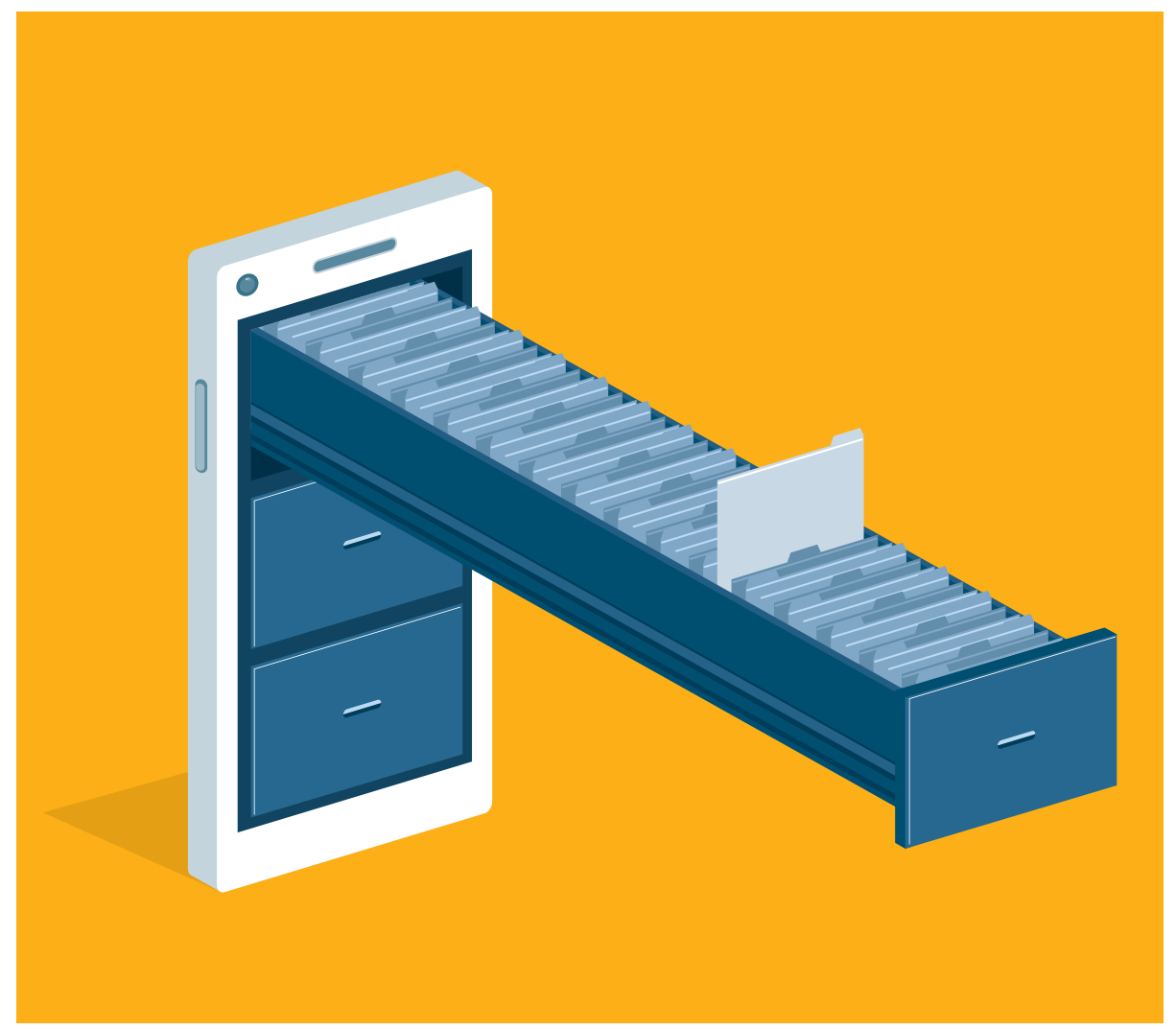

Credit: sorbetto / DigitalVision Vectors / Getty

sex development ${ }^{4}$. Data should be collected in EHRs in the context of community needs and organizational capacity assessments, integrated service model development, treatment protocol adoption and trauma-informed approaches 5 .

Healthcare organizations can identify internal staff champions from administrative, clinical, registration and information technology departments to collaboratively delineate implementation timelines and protocols ${ }^{6}$. Plan-Do-StudyAct cycles, and other continuous quality improvement methods, enable iterative enhancements in data collection efficiency and effectiveness. Tailoring of standardized sexual orientation and gender identity question stems and response options in collaboration with local communities, for both routine demographic registration forms and clinical encounters, can create a more culturally and linguistically resonant patient experience.

Data collection for entry into EHRs may occur via paper forms, electronic tablets or telehealth modalities, and correct names and pronouns can be linked to laboratory testing and electronic medication prescribing orders ${ }^{7}$, as for HIV pre-exposure prophylaxis (PrEP) or gender-affirming hormone therapy. Organizations with more limited internal information technology design capabilities can inquire whether their EHR vendor has pre-existing sexual orientation 


\section{Table 1 | EHRs for equity}

\section{Current EHR data applications for LGBTQIA+ equity}

Displaying correct patient name and pronouns through banners, alerts, automated salutations, letter templates, laboratory tests, prescriptions and patient instructions

Anatomical inventories for clinical decision support

Population health management to reduce disparities in primary care quality outcomes

Delivery of gender-affirming psychosocial, medical and surgical care

Tailored sexual health counseling regarding SARS-CoV-2 transmission risks

\section{Emerging and potential EHR data applications for LGBTQIA+ equity}

Addressing disparities in SARS-CoV-2 testing, infection, health outcomes and vaccination

Preventing medication-induced teratogenicity for people who can become pregnant and do not identify as female (e.g., isotretinoin registry)

Identifying patients who are candidates for HIV pre-exposure prophylaxis

Improving global HIV epidemic control

Improving care for people with differences of sex development and intersex variations

and gender identity customization options to suit their unique workflows. Possible features include EHR banners, alerts, automated salutations, letter templates and patient instructions that effectively display correct names and pronouns in each context ${ }^{6}$.

\section{Patient-centered care}

EHRs that arbitrarily associate patients' registration sex with presumed organs can cause clinical errors and insurance reimbursement denials for necessary interventions and preventative screenings, such as failing to offer or pay for routine cervical Pap tests with trans masculine patients who retain a cervix ${ }^{8}$. Instead, EHR anatomical inventories allow care teams to note and track retained organs and body modifications; in turn, anatomical inventories can synergize with clinical decision support algorithms to guide patient-centered primary and preventive care.

There are numerous current applications of EHR data to improve care for LGBTQIA+ people (Table 1). Gender identity information and anatomical inventories can facilitate developmentally appropriate delivery of gender-affirming psychosocial, pharmacological and surgical interventions that are associated with better mental health outcomes, including lower suicidality ${ }^{9-11}$. Knowledge of patients' sexual orientation identity and behavior is important for effective counseling regarding SARS-CoV-2 transmission risks $^{12}$. Collection of patient sexual orientation and gender identity data has enabled the identification of disparities within healthcare organizations, including in rates of cervical cancer screening among cisgender sexual minority women compared to cisgender straight women, and among transgender men compared with cisgender women ${ }^{13}$.

\section{Health equity}

Emerging opportunities for culturally responsive data collection hold promise for further advancing LGBTQIA+ health equity (Table 1 ), for example by improving understanding of and reducing disparities related to SARS-CoV-2 in testing, infection, outcomes and vaccination among sexual and gender minority populations experiencing adverse social determinants of health ${ }^{14}$. In the future, incorporating gender identity data collection during isotretinoin prescription registration for treating severe acne could prevent teratogenicity among people who can become pregnant and do not identify as female ${ }^{15}$.

Innovative prediction algorithms are starting to link EHR information identifying men who have sex with men to data on sexually transmitted infection positivity and other HIV risk factors, and then applying machine learning to more effectively identify potential candidates for $\operatorname{PrEP}^{16}$. Sexual orientation and gender identity data collection is a key facilitator of improving quality of care and HIV epidemic control for sexual and gender minority communities globally, including in sub-Saharan Africa. This can occur within screening protocols led by health departments as a means to identify patients most at risk for HIV and therefore in need of being prioritized for $\operatorname{PrEP}^{17}$. Likewise, developing validated demographic questions pertaining to differences of sex development and intersex variations (DSDI), in partnership with people who have DSDI, will facilitate delivery of sensitive and effective care ${ }^{18}$.

\section{Training and policy}

A critical parallel effort to data collection initiatives is training of the healthcare workforce. Most clinicians do not discuss sexual orientation or gender identity with patients, owing to a belief that this information lacks relevance to care, concern about causing patient discomfort or offense, and a lack of the clinical experience, knowledge and language to have these conversations $^{19}$. Over the past decade, education in sexual and gender minority health has increasingly expanded toward reaching all healthcare professionals, including through mandatory trainings $\mathrm{s}^{20}$. Clinician education ought to emphasize mastery of basic concepts related to sexual orientation, gender identity and sex development, sensitive and effective communication with LGBTQIA+ patients, and building inclusive and affirming healthcare environments for LGBTQIA+ communities ${ }^{4}$. All healthcare staff should be required to have training to mitigate the adverse impact of implicit bias against patients who are members of sexual and gender minorities ${ }^{21}$.

Inclusive guidelines and protective policies at national, regional and organizational levels can yield transformative benefits. Since 2016, the US Health Resources and Services Administration's Bureau of Primary Health Care has required all federal qualified health centers, serving almost 30 million people in the United States, to report patient sexual orientation and gender identity data on an annual basis ${ }^{22}$. Implementation of this policy enabled several healthcare organizations to detect disparities based on sexual orientation, gender identity or both in screening rates for cervical cancer, depression and tobacco use ${ }^{13}$. US city-level sexual orientation and gender identity nondiscrimination laws are associated with more complete sexual orientation and gender identity data in EHRs at federally qualified health centers ${ }^{23}$. This finding suggests that federal nondiscrimination laws protecting LGBTQIA+ people, such as the Equality Act passed by the US House of Representatives in February 2021 and under consideration in the Senate at the time of writing, could help achieve more uniform patient data completeness across the country, as well as the identification of additional disparities for local, state and national public health intervention.

\section{Lived experience}

Through their lived experience, people with diverse sexual orientations, gender identities and sex development are uniquely poised to guide EHR-related technology, protocol and 
policy design that addresses LGBTQIA+ health inequities. Financially compensated inclusion of LGBTQIA+ people ought to occur in three important settings ${ }^{24}$. The first consists of healthcare-associated community advisory boards that meaningfully influence organizational leadership and service delivery for meeting population-specific needs. The second is medical coding boards that inform administrative classification and billing for health services. Finally, representation of LGBTQIA+ people within health data standards development organizations, such as the Health Level Seven International Gender Harmony Project ${ }^{25}$, will facilitate the ongoing refinement and scale-up of interoperable, high-quality and culturally responsive EHR systems in the United States and across the globe.

Alex S. Keuroghlian (1) 1,2,3凶

${ }^{1}$ The Fenway Institute, Fenway Health, Boston,
Massachusetts, USA. ${ }^{2}$ Massachusetts General Hospital, Boston, Massachusetts, USA. ${ }^{3}$ Harvard Medical School, Boston, Massachusetts, USA.

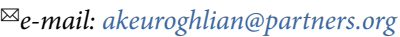

Published online: 22 November 2021

https://doi.org/10.1038/s41591-021-01592-3

\section{References}

1. National Academies of Science, Engineering, and Medicine. https://www.nationalacademies.org/our-work/understanding-the status-and-well-being-of-sexual-and-gender-diverse-populations (accessed 11 September 2021)

2. Cahill, S. et al. PLoS One 9, e107104 (2014).

3. Loo, S. et al. PLoS One 16, e0255568 (2021).

4. Goldhammer, H. et al. J. Health Care Poor Underserved 32, 18-29 (2021).

5. Morenz, A. M. et al. Ann. Fam. Med. 18, 73-79 (2020).

6. Grasso, C. et al. J. Am. Med. Inform. Assoc. 26, 66-70 (2019).

7. Grasso, C. et al. Transgend. Health https://doi.org/10.1089/ trgh.2020.0155 (2021)

8. Grasso, C. et al. J. Am. Med. Inform. Assoc. 28, 2531-2535 (2021).

9. Almazan, A. N. et al. JAMA Surg. 156, 611-618 (2021).

10. Turban, J. L. et al. Pediatrics 145, e20191725 (2020).

11. Turban, J. L. et al. J. Adolesc. Health https://doi.org/10.1016/ j.jadohealth.2021.06.001 (2021).

12. Turban, J. L. et al. Ann. Intern. Med. 173, 387-389 (2020).
13. Grasso, C. et al. Int. J. Med. Inform. 142, 104245 (2020). 14. Cahill, S. et al. Am. J. Public Health 110, 1360-1361 (2020). 15. Singer, S. et al. LGBT Health 7, 216-219 (2020). 16. Marcus, J. L. et al. Lancet HIV 6, e688-e695 (2019). 17. Keuroghlian, A. S. et al. J. Int. AIDS Soc. 24, e25728 (2021).

18. National LGBTQIA+ Health Education Center at The Fenway Institute. https://www.lgbtqiahealtheducation.org/publication/ affirming-primary-care-for-intersex-people-2020/ (accessed 11 September 2021)

19. Goldhammer, H. et al. LGBT Health 5, 461-468 (2018). 20. Ard, K. L. et al. N. Engl. J. Med. 379, 2388-2391 (2018). 21. McDowell, M. J. et al. Psychosomatics 61, 655-661 (2020) 22. Grasso, C. et al. Am. J. Public Health 109, 1111-1118 (2019) 23. Almazan, A. N. et al. Am. J. Public Health, e1-e5 (2021).

24. Kronk, C. A. et al. J. Am. Med. Inform. Assoc. https://doi.org/ 10.1093/jamia/ocab136 (2021)

25. McClure, R. C. et al. J. Am. Med. Inform. Assoc. https://doi.org/ 10.1093/jamia/ocab196 (2021).

Acknowledgements

A.S.K. thanks the advocates, clinicians, scientists, technologists, educators and policymakers whose efforts promote LGBTQIA+ health equity.

Competing interests

A.S.K. stands to receive future royalties as editor of a McGraw-Hill textbook on transgender and gender diverse healthcare. A.S.K. has no other competing interests. 